\title{
Towards Distributed Transmission Scheduling for Wireless Ad Hoc Networks
}

\author{
Angelos Vassiliou, Themistoklis Charalambous, Ioannis Krikidis and Evelina Klerides
}

\begin{abstract}
In this paper we study distributed transmission scheduling via power control in wireless ad hoc networks with multiple channels. The target for each node is to manage to be admitted into a channel from the available channels in the network. The aim of this work is twofold: (a) to determine how a wireless node, based on its limited information, will decide which channel to access and (b), to propose a distributed algorithm for each wireless node with which once the channel is chosen a decision is made whether to stay in the channel or not. Here, we propose an algorithm that, if adopted by all the nodes in the network, it converges to a solution that admits most of the wireless nodes in the network, based on limited information only. Simulations in MATLAB justify the good performance of the algorithm.
\end{abstract}

\section{INTRODUCTION}

Wireless networks in general have attracted tremendous attention both from academia and industry. As a result, a wide variety of applications for wireless networks are emerging, being patented and commercialized. Furthermore, with the widespread use of multimedia application with Quality of Service (QoS) demands, there is a lot of ongoing research on how to provide reliable QoS support to these networks.

In wireless networks, communicating nodes are usually equipped with half-duplex transceivers. This implies that at any point in time each node is limited to transmit only on a single channel of fixed range of frequency spectrum. In large networks there exist inevitably many transmissions and it is therefore unlikely that a channel will be used only by a single wireless node. The main motivation of this work is Orthogonal Frequency Division Multiple Access (OFDMA) where wireless nodes share some common channels and can be scheduled accordingly.

Each transmission corresponds to a spatiotemporal propagation of radio waves that are received by all nodes in proximity using the same channel simultaneously. Hence, nodes interfere and the level of interference depends on the received signals from all transmitters in the channel at the time. The higher the transmitting power from irrelevant nodes in the same channel, the higher the interference experienced at a receiver.

Even though the computation of efficient schedules in the abstract physical model with power control has been extensively studied (for example in [1]-[5]), its problem is proven to be NP-hard [6], given that we know minimum $\left(P_{\min }>0\right)$

Angelos Vassiliou, Themistoklis Charalambous and Ioannis Krikidis are with the Department of Electrical and Computer Engineering, University of Cyprus, Nicosia, Cyprus. E-mail: \{angelos.vassiliou, themis, krikidis\}@ucy.ac.cy.

Evelina Klerides is with the Business School, Imperial College London, UK. E-mail: e.klerides05eimperial.ac.uk. and maximum $\left(P_{\max }<\infty\right)$ transmission power levels.Also, since centralized transmission scheduling schemes suffer from the large-scale data dissemination and management and incur network vulnerability, distributed schemes have become particularly attractive. A successful distributed algorithm requires only local measurements for decision making and updating, so that eventually all wireless links (or at least as many as possible) meet their QoS requirements, i.e., the solution converges to an optimal or sub-optimal solution.

This work concerns scheduling data traffic in a distributed fashion of unicast applications sharing the ad hoc network under the abstract physical model. Such applications are naturally mapped to the point-to-point single-hop traffic model, where the entities to be scheduled are links. This problem, within the physical model framework, has been targeted before, such as [7], [8] and [9]. In these approaches, however, a suite of Distributed Power Control algorithms with Active Link Protection (DPC/ALP) schemes is presented capturing the essential dynamics of power control. Even though this schemes protect nodes from transmitting at their maximum power, preventing them from draining their battery while adding to the interference only, they consider only access to a single channel and in addition, they reduce the network's performance by allowing in many cases "weak" communication pairs to monopolize the wireless channel favoring links that are activated earlier than others, hence inhibiting some communication pairs to establish a connection. Our algorithm combines (a) an approach on how to access one of the available channels, (b) how to proceed once a channel has been chosen and (c) how to behave once it is part of a channel and other nodes try to get access.

The rest of the paper is organized as follows. In Section II we describe the employed system model and in Section III it is derived when a network is considered feasible. Section IV provides the formulation for the problem being targeted. Section V thoroughly describes the distributed algorithm for distributed transmission scheduling. Section VI evaluates the performance of our methodology. Finally, we close in Section VII, where we draw conclusions and give directions for future work.

\section{SySTEM MODEL}

The system model can be divided into two levels: the network as a whole and an individual channel. Thus, we distinguish between the network and the channel model. The network model concerns the general topology of the nodes and their characteristics. The channel model describes the 
assessment of the link quality between communication pairs and the interaction between the nodes in the network.

In this study, we consider a network where the links are assumed to be unidirectional and each node is supported by an omnidirectional antenna. For a planar network (easier to visualize without loss of generality), this can be represented by a graph $\mathcal{G}=(\mathcal{N}, \mathcal{L})$, where $\mathcal{N}$ is the set of all nodes and $\mathcal{L}$ is the set of the active links in the network. Each node can be a receiver or a transmitter only at each time instant due to the half-duplex nature of the wireless transceiver. Each transmitter aims to communicate with a single node (receiver) only, which cannot receive from more than one node simultaneously. We denote by $\mathcal{T}$ the set of transmitters and $\mathcal{R}$ the set of receivers in the network.

The link quality is measured by the Signal-to-interferenceand-Noise-Ratio (SINR). The channel gain on the link between transmitter $i$ and receiver $j$ is denoted by $g_{i j}$ and incorporates the mean path-loss as a function of distance, shadowing and fading, as well as cross-correlations between signature sequences. All the $g_{i j}$ 's are positive and can take values in the range $(0,1]$. The power level chosen by transmitter $i$ is denoted by $p_{i}$ and the intended receiver is also indexed by $i . \nu_{i}$ denotes the variance of thermal noise at the receiver $i$, which is assumed to be additive Gaussian noise. The interference power at the $i^{\text {th }}$ node, $I_{i}$, includes the interference from all the transmitters in the network and the thermal noise, and is given by $I_{i}=\sum_{j \neq i, j \in \mathcal{T}} g_{j i} p_{j}+\nu_{i}$. Therefore, the SINR at the receiver $i$ is given by

$$
\Gamma_{i}=\frac{g_{i i} p_{i}}{\sum_{j \neq i, j \in \mathcal{T}} g_{j i} p_{j}+\nu_{i}} .
$$

Due to the unreliability of the wireless links, it is necessary to ensure Quality of Service (QoS) in terms of SINR in wireless networks. Hence, independently of nodal distribution and traffic pattern, a transmission from transmitter $i$ to its corresponding receiver is successful (error free) if the SINR of the receiver is greater or equal to the capture ratio $\gamma_{i}$ ( $\Gamma_{i} \geq \gamma_{i}$ ), which depends on the modulation and coding characteristics of the radio. Therefore,

$$
\frac{g_{i i} p_{i}}{\sum_{j \neq i, j \in \mathcal{T}} g_{j i} p_{j}+\nu_{i}} \geq \gamma_{i}
$$

\section{FEASIBILITY OF A NETWORK} ing

Equation (2) after manipulation, is equivalent to the follow-

$$
p_{i} \geq \gamma_{i}\left(\sum_{j \neq i, j \in \mathcal{T}} \frac{g_{j i}}{g_{i i}} p_{j}+\frac{\nu_{i}}{g_{i i}}\right) .
$$

In matrix form, for a network consisting of $n$ communication pairs, this can be written as

$$
\mathbf{p} \geq \Gamma G \mathbf{p}+\boldsymbol{\eta}
$$

where $\Gamma=\operatorname{diag}\left(\gamma_{i}\right), \mathbf{p}=\left(\begin{array}{llll}p_{1} & p_{2} & \ldots & p_{n}\end{array}\right)^{T}, \eta_{i}=$ $\gamma_{i} \nu_{i} / g_{i i}$, while $G_{i j}=g_{j i} / g_{i i}$ if $i \neq j$, and zero otherwise. Let $C=\Gamma G$, so that (4) can be written as $(I-C) \mathbf{p} \geq \boldsymbol{\eta}$. The matrix $C$ has nonnegative elements and it is reasonable to assume that is irreducible, since we are not considering totally isolated groups of links that do not interact with each other. By the Perron-Frobenius theorem [10], we have that the spectral radius of the matrix $C$ is a simple eigenvalue, while the corresponding eigenvector is positive componentwise. The necessary and sufficient condition for the existence of a nonnegative solution to inequality (4) for every positive vector $\boldsymbol{\eta}$ is that $(I-C)^{-1}$ exists and is nonnegative. However, $(I-C)^{-1} \geq 0$ if and only if $\rho(C)<1$ [11] (Theorem 2.5.3), where $\rho(C)$ is the spectral radius of $C$.

\section{Problem Formulation}

The problem being targeted here is the following: Given a set of available channels and a set of communicating pairs comprised by wireless nodes that have limited information about the available channels, what should be the policy adopted by each node such that a feasible network is eventually achieved?

In order to formulate the optimization problem, we define two sets of decision variables, for each transmitter $i \in \mathcal{T}$ and channel $t=1, \ldots, D$ ( $D$ is the maximum number of available channels); processing-time variables:

$$
x_{i}(t)= \begin{cases}1, & \text { if transmitter } i \text { is active at time } t \\ 0, & \text { otherwise }\end{cases}
$$

and power level variables: $p_{i}(t) \in \mathbb{R}_{+}$.

Since the problem involves both integer and continuous decision variables, the mathematical formulation is classified as a Mixed Integer Program (MIP) and is given in Model 1.

\begin{tabular}{l}
\hline Model 1 Problem formulation \\
\hline $\begin{array}{c}\text { minimize } \\
\text { x,p } \\
\text { subject to }\end{array}(\mathbf{x}, \mathbf{p})$ \\
$\sum_{t=1}^{D} x_{i}(t) \geq 1 \forall i \in \mathcal{T}$, \\
$x_{i}(t)=0 \Rightarrow p_{i}(t)=0 \forall i \in \mathcal{T}, t=1, \ldots, D$, \\
$x_{i}(t)=1 \Rightarrow g_{i i} p_{i}(t) \geq \gamma_{i}\left(\sum_{j \in \mathcal{T}, j \neq i} g_{j i} p_{j}(t)+\nu_{i}\right)$ \\
$\forall i \in \mathcal{T}, t=1, \ldots, D$, \\
$x_{i}(t) \in\{0,1\} \forall i \in \mathcal{T}, t=1, \ldots, D$, \\
$p_{i}(t) \in \mathbb{R}_{+} \forall i \in \mathcal{T}, t=1, \ldots, D$.
\end{tabular}

The transmission scheduling problem may have one or more optimal solutions and the solutions vary according to the optimization problem being targeted; i.e., it depends on the minimizing function $f(\mathbf{x}, \mathbf{p})$. If, for example, the target was to minimize the total power in the network at every time instant $t$, then

$$
f(\mathbf{x}, \mathbf{p})=\sum_{t=1}^{D} \sum_{j \in \mathcal{T}} x_{j}(t) * p_{j}(t)
$$

It is obvious that there is no way to ensure convergence to an optimal solution of a transmission scheduling problem 
in a distributed fashion, when there is only limited partial information about the whole network.

A possible approach could be that the best channel for a node to use is the channel that causes the least interference to that particular node. However, this approach may cause problems due to the following reason: It is possible that a node senses little interference from other communication links in a certain channel, but some nodes in that channel experience a lot of interference from that node. In this case, that node, lets call it $A$, can potentially enter the channel, causing interference to other nodes, high enough to force them abandon the channel. In such a case, Active Link Protection (ALP) inherent to our proposed algorithm disappears, because an established link will be forced to leave the channel so that node $A$ 's link is established. In order to guarantee some form of ALP, a protection mechanism should be devised.

Furthermore, it can be easily seen that such an approach may not lead to an optimal solution. This can be seen via an illustrative example: Suppose that node $A$ can potentially be activated using any of the channels 1, 2 or 3, without any nodes already active leaving the channels. Also, suppose that in all optimal solutions, nodes $A$ and $B$ have to be in the same channel. If node $B$ is already active in channel 3 , yet channel 1 produces the minimum interference to node $A$, then node $A$ would choose to join channel 1, diverging from the optimal solution. Furthermore, suppose on top that nodes $A$ and $C$ cannot both become active (reach the capture ratio $\gamma_{i}$ simultaneously) in the same channel. If nodes $B$ and $C$ can reach the capture ratio $\gamma_{i}$ simultaneously in the same channel and are currently active in a channel (say channel 3), then we can consider the following scenarios:

- minimum interference is sensed from channel 1. As a result, channel 3 will never be chosen by node $A$, with the network diverging from the optimal solution;

- minimum interference is sensed from channel 3. As a result, channel 3 will be chosen. Then, either $A$ or $C$ will have to abandon the channel. The question is how it is decided which node to abandon the channel. If we decide that the one that reaches its maximum power needs to leave, then the question becomes: which one node reaches $p_{\max }$ faster? It is quite possible that node $A$ will reach $p_{\text {max }}$ faster than node $C$, meaning that node $A$ will never stay in the same channel with $B$, thus never achieving the optimal solution.

\section{Proposed ALgORithm}

\section{A. Algorithm overview}

The basis for our proposed power control algorithm is the Foschini-Miljanic (FM) algorithm [12]. The FM algorithm requires that transmitters be aware of the channel state to the various possible receivers. However, Channel State Information (CSI) is not automatically available at the transmitters, but it has to be acquired. When a node enters a channel, it attempts to converge to the optimal power level using the FM algorithm. If the node's power at any time instant reaches a value greater than $p_{\max }$, then the node exits the channel.
This means that it is possible for a communication pair (with an established link) to abandon a channel when a new node is admitted into the channel and causes much interference, more than what the transmitter of the established link could handle. This could be considered as a drawback if Active Link Protection (ALP) is a priority. However, in this work, we assume that bursty traffic over bursty links [13] is also possible and ALP is not a big issue.

Whenever a node leaves a channel, it will start measuring the following characteristics (that we call channel's characteristics for each node): (a) Channel's interference sensed at the receiver: interference should only be measured when a channel's interference is stable over a period of time. While a node's power level in a candidate channel has not yet converged to its optimal power level, the sensed interference could be variable. The transient interference experienced by the receiver when the transmitter is silent does not provide any information about the channel, but only about the convergence process. In order to determine whether a channel's interference is stable over a period of time $k$, its standard deviation should be smaller than a certain value $\epsilon$; i.e., $\sigma(I(n-k) \ldots I(n)) \leq \epsilon$. (b) Channel's rate of change of interference $(\Delta I) . \Delta I$ for a node $i$ is defined as $\Delta I_{i}(n)=I_{i}(n)-I_{i}(n-1)$, given that $p_{i}(n-2)=0$, and $p_{i}(n-1)=p_{\text {trial }}$. It is necessary that the channel's interference is stable before starting the process of probing the channel. The probing occurs by a node transmitting a probing signal with power $p_{\text {trial }} . \Delta I$ is measured after a channel with stable interference is probed. During that process, the channel reacts in a specific way, and the reaction is measured. It is easy to realize that during the probing and the period to converge back to the steady state conditions, individual node's SINRs drop below their capture ratio $\gamma_{i}$. This is not a problem, because we can set the capture ratio to have a "safety" margin above the minimum value of SINR for which the messages are decoded error free.

In addition, we define a metric which will be used to help nodes decide which channel to enter. We define $Q=I \times$ $\Delta I$ to denote the product of the channel's interference and rate of change of interference. After the measurements have been taken $(I$ and $\Delta I)$ a node selects a candidate channel to join. The way the channel decides when to enter the network is described later. An overview of our proposed algorithm is shown in Figure 1.

\section{B. Adaptation of the FM algorithm}

We use the FM algorithm, but nodes behave in a specific manner when the FM-dictated power becomes $p_{\mathrm{FM}}>p_{\max }$. During the measurement stage of each node, nodes use socalled probes to measure the channel's reaction to a small transmission from the measuring node. It is possible that a node has converged to a specific power in a channel that is probed, to a power level close to $p_{\max }$. During the fleeting transmission of a node that is just probing the channel, this node using the FM algorithm will determine that its power should become greater than $p_{\max }$, to compensate for the interference caused by the probing node. We wish to protect nodes 


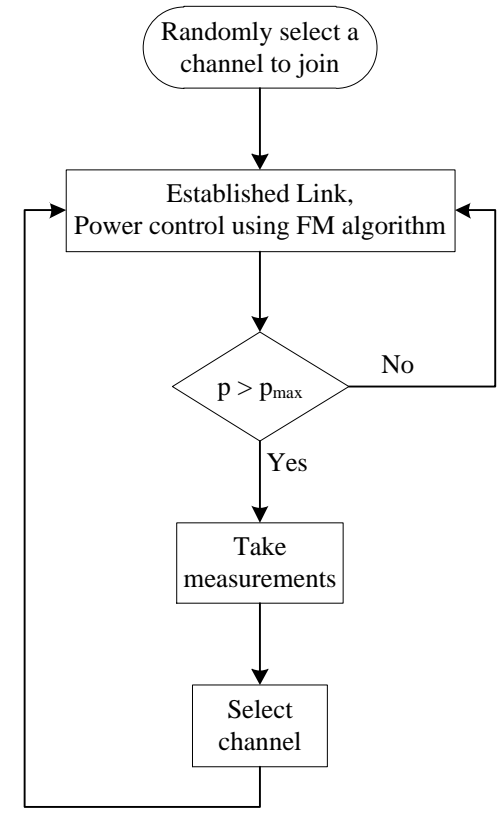

Fig. 1. An overview of the proposed algorithm: Once a node is in a channel, it will use the FM algorithm to determine its transmitting power. If this power becomes greater than $p>p_{\max }$, the node will leave its current channel. After the node leaves the channel it will take measurements of different channel characteristics, for all channels. Then using these measurements, (or not), the node will select a channel to join. The node will next join the selected channel, and once again use the FM algorithm to attempt to converge in the selected channel.

from such temporary spikes in interference. Our proposed adaptation is simply a counter that allows a node to reach $p_{\text {max }}$ for just a limited time, and if the increased interference subdues, then the node may remain in the channel. Otherwise, probing could potentially kick nodes out of channels that they would otherwise be just fine in. In the case that the increased interference persists, then it is obvious that it is not caused by a temporary probe, and so the node will abandon the channel.

\section{Measuring channel's characteristics}

Besides measuring interference $I$ in a channel, measuring a channel's $\Delta I$ also poses some challenges. One issue with measuring $\Delta I$ accurately is that several nodes might concurrently probe the channel, with the channel reacting to both probes, providing an inaccurate $\Delta I$ to both nodes. In order to avoid this, we implemented a random wait counter on each node, with each node waiting a random amount of time after a channel stabilizes, to probe it. The statistical chances of two nodes probing simultaneously occurring are low, but to avoid even such a case, measurements are taken three times for each channel, and if all three times the measurements are equal they are accepted and used for decision making.

\section{Selection of a channel}

We separate the Channel selection algorithms into two groups. The random group and the deterministic group.

The random group includes all the channel selection algorithms that select what channel to join randomly; either totally randomly or by using weights in their random selections. These schemes are as follows: (i) Fully random selection: In this scheme, no measurements are taken into account, but each node tries to enter a channel based on a uniform distribution on the probability of choosing between the available channels; (ii) random selection with $I_{i}^{-1}$ being the weight for each node $i$; i.e., the larger the interference experienced at the receiver, the smallest the weight and hence the probability of selecting the specific channel; (iii) Random selection with $Q_{i}^{-1}$ being the weight for each node $i$; i.e., the larger the value of $Q_{i}$, the smallest the weight and hence the probability of entering the specific channel.

The deterministic group includes the algorithms that select a channel to join based merely on measurements; the channel is selected by choosing the minimum measured quantity or metric: (a) $\min (I)$, (b) $\min (\Delta I)$, and (c) $\min (Q)$. However, for the deterministic group, once a node selects a channel that it cannot join on its next attempt, if nothing has changed, it will once again select the same channel to attempt to join, since it is the best channel to enter according to the measurements. As a result, the node will attempt to enter the selected channel and will once again fail to do so.

\section{PERformance EVAluation}

For our computational study we generated a set of test networks of various sizes which will serve as the benchmark for evaluating our algorithm. More specifically, the benchmark set consists of networks with $10,20,30, \ldots, 100$ pairs (we have 10 instances for each size) and hence, in total, we have a test set consisting of 100 networks. Each node made decisions based solely on its available local information. We then compared the number of active nodes at the end of the simulations.

\section{A. Performance measures}

We examined the percentage of nodes that managed to reach the capture ratio $\gamma_{i}$ and have converged to a transmitting power within a channel. We ran the simulations for our 100 networks, and calculated the average percentage of active nodes for all networks. We then ran our simulations for a different number of channels, and for each of our channel selection algorithms. For each network we found the optimal (minimum) number of channels, such that all wireless links can operate simultaneously and used that as our starting point for selecting the number of channels for our simulations. We run our simulations for the optimal, optimal+1 and optimal+2 number of channels.

\section{B. Example}

In the presented simulation we will see the power levels of two transmitters, and how their SINR varies with time. The network in question has a size of $N=50$, and has 4 channels. The capture ratio $\gamma_{i}$ is equal for all nodes in the network, and is $\gamma_{i}=3$.

At time 600, node 44 is already using channel 4 for its communications. Node 39 is not in any channel at time 600, 

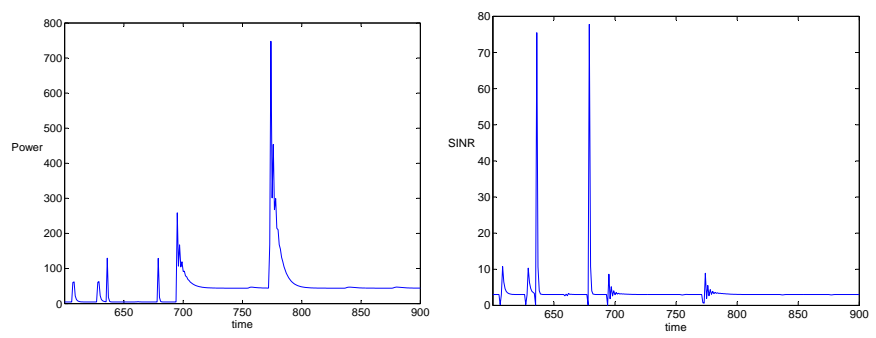

Fig. 2. The transmitter's power and SINR levels for node 44 , for a simulated time period.

but is in the "take measurements" stage at that point. In this example we see the changes of the two node's power levels and SINRs for a period of time, namely for the simulated time between 600 and 900. In Fig. 2 (left) we can see the power level for node 44. From the time period of 600 to 694, node 44's power is lower than the power level after $t=694$. This shows us that a new node entered node 44 's channel at $t=694$. At $t=608,628,636,697$ we notice that there are some spikes in node 44 's power. These spikes are caused by some other node's probing of channel 4 . Visually we can conclude that the same node caused the same disturbance at $t=608$ and $t=628$, because node 44's reaction to these two disturbances is identical. A different node caused the disturbances at $t=636$ and $t=697$. Since the probes only last for 1 cycle, their effects quickly diminish. The network converges to the optimal power using the FM algorithm. At $t=694$, node 39 decides to join channel 4 , thus causing a new disturbance in the network. The new node apparently joined the channel using an initial power that is bigger than the optimal power. That is why the FM algorithm causes all nodes in channel 4 to lower their powers until the new optimal power is reached $\left(p_{44}=43.87\right.$ at $\left.t=737\right)$. In Fig. 2 (right) we can see node 44's SINR as it varies during the $t \in[600,900]$ time frame. During this time we can see some dips in SINR for very short periods of time (just a single cycle), followed by a sharp rise in SINR. This is the typical reaction of the FM algorithm in case of a single cycle increase in interference, such as a probe. During the probe's duration, the interference sensed by node 44 increases sharply, and so the FM algorithm dictates that the node increases its power. As soon as the power is raised in the following cycle, the cause of the interference vanishes, and this means that the SINR will rise above $\gamma_{i}$. Even though there are often probes, we can see that the FM algorithm allows node 44 to quickly converge to an $\operatorname{SINR}=\gamma_{i}$. These frequent (temporary) drops in SINR are a main disadvantage of an algorithm that relies on probing channels.

In Fig. 3 (left) we can see node 39's power level over time. Our first observation is the spike in power at $t=690$. This spike has a height of exactly $p_{39}=92$, which is equal to this node's probing power. The spike is a probe that was transmitted at channel 3 . That probe actually concluded the "take measurements" stage for node 39. During the next cycle $(t=691)$, node 39 measured channel 3's reaction to its
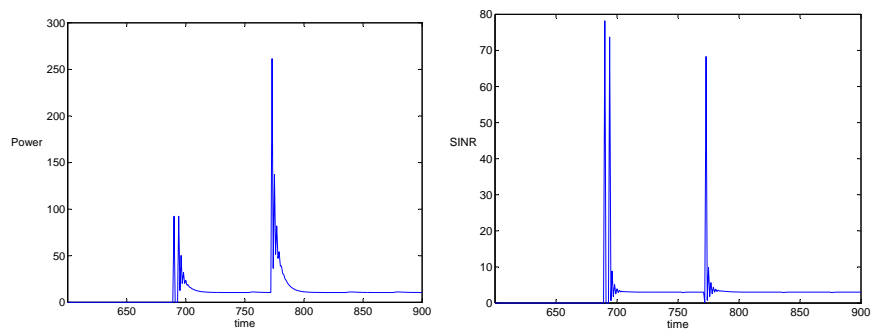

Fig. 3. The transmitter's power and SINR levels for node 39, for a simulated time period

probe. At $t=692$, node 39 performed the Channel Selection algorithm $(\min (\Delta I)$ in this particular example), and channel 4 was selected. At $t=694$, node 39 joined channel 4 with $p_{39}(694)=92$, which proved greater than the optimal power, with node 39 converging to its optimal power by $t=731$, at $p_{39}(731)=10.5$. In Fig. 3 (right) we can see node 39's SINR as it varies during the $t \in[600,900]$ time frame. During this time we can see three major events. At $t=690$, node 39's SINR rises sharply because a probe will abruptly (and unsustainably) increase the SINR. Since node 39 stops transmitting in channel 3 after the probe, its SINR drops to 0 . Once it joins channel 4 at $t=694$, its SINR is initially high (due to the high initial power), but the FM algorithm quickly controls node 39's power so that node 39 converges to $\Gamma_{39}=\gamma_{39}=3$. At $t=773$, we can see node 39's SINR reacting to an event at $t=772$, just like node 44 reacts in Fig. 2. This makes sense, since both nodes are using the same channel.

In both Figures 2 and 3, we can see the common reaction to a disturbance at $t=773$. At this time, a node probed channel 4. We can see that the degree of reaction depends on $g_{i j}$, and since $g_{i 44} \neq g_{i 39}$, the two node's degree of reaction differs. The node probing channel 4 will at this time have to discard any earlier measurements, since channel 4's reaction to probes has now changed, due to the additional nodes reacting to the probe.

\section{Results}

From Fig. 4 we can see that the network size also affects the number of active nodes. But since the network's size does not monotonically affect the percentage of active nodes, nor does it affect the percentage significantly, we can deem $N$ as irrelevant to an algorithm's performance. What increases the percentage of active nodes is the number of channels. We can see that our algorithms will allow most data links to become active. This is a good threshold for comparing algorithms. We can also see that there might be little benefit of adding another channel into the network, just to satisfy an extra number of links.

We can see the results from our simulations in table I. From the table we can compare the performance of proposed algorithms. The similarity of the results of the algorithms when compared to each other is interesting. We can also see that the average "success" rate is pretty high. The performance of all 


\begin{tabular}{l|c|c|c} 
Number of Channels & Optimal & Optimal+1 & Optimal+2 \\
\hline \hline Algorithm & & & \\
Random Channel & 92.08 & 97.85 & 99.61 \\
R.W. Interference & 92.50 & 97.44 & 99.46 \\
R.W. $Q$ & 92.88 & 97.72 & 99.17 \\
$\min (I)$ & 91.69 & 96.70 & 98.44 \\
$\min (\Delta I)$ & 92.43 & 97.68 & 99.17 \\
$\min (Q)$ & 92.60 & 97.05 & 98.94
\end{tabular}

TABLE I

PERCENTAGE OF NODES ACTIVE AT THE END OF THE SIMULATION

examined algorithms improves significantly with the number of channels, but these number hide one important factor. With various algorithms we also get varying degrees of ALP. The more random an algorithms is the less the ALP it provides. This means that using the fully random algorithm (where a channel is chosen at random) will yield the best results, but some nodes will not be stable in or out a channel for long periods in the network.

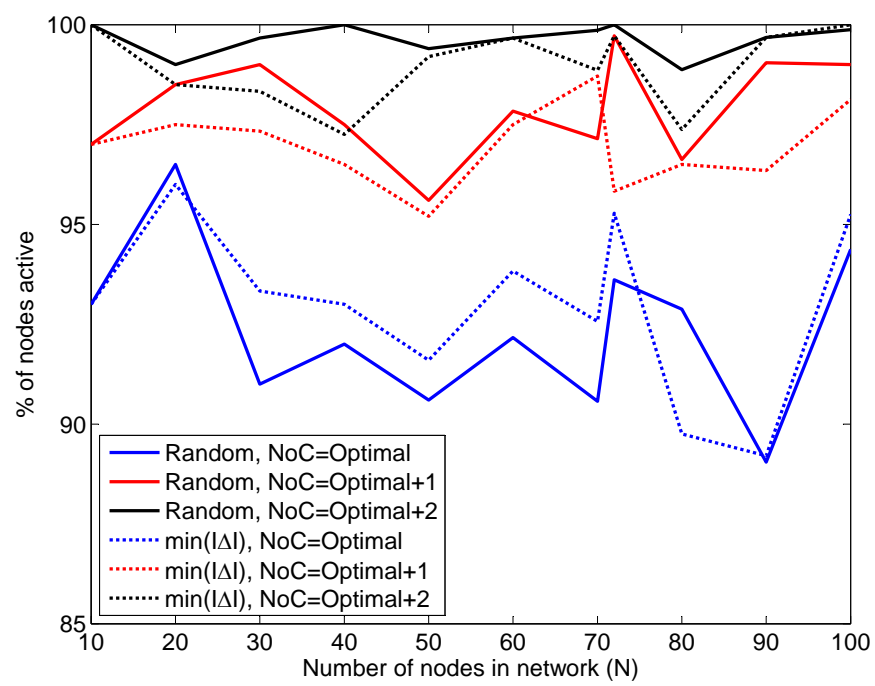

Fig. 4. How the number of channels affect the number of nodes that become active in a channel. The dotted lines represent the active nodes when using the $\min (Q)$ selection scheme.

In the Fig. 4, we can confirm that the Random selection algorithm works slightly better when the number of channels is large, yet the more deterministic $\min (Q)$ algorithm works better when the number of channels is low. The deterministic algorithms actually provide more ALP and yields better results in a congested network with fewer channels in contrast to the random selection algorithm that gives better results when the number of channels is greater than the optimal number of channels. It seems that the two algorithms offer a trade-off between node complexity, ALP, and the percentage of nodes that converge to an active communication mode.

The random selection method is certainly the simplest to implement, and causes the least side effects (since it lacks any probing functions). It also requires the least overhead since no feedback information has to be communicated during the probing process. The $\min (Q)$ selection scheme seems to perform slightly better for a smaller number of channels, and since this is often the case, it means that there might be some merit to using the $\min (Q)$ selection scheme.

\section{Conclusions And Future Directions}

We developed a distributed algorithm with which each node selects, enters a channel from a number of available channels. From the examined channel selection methods, the uniformly random selection method seems to offer the best results, with any other methods either trailing behind, or negligibly outperforming it. Due to the random method's simplicity and the considerable reduction in communication overhead, it seems that it is the indicated solution for OFDMA networks. The distributed algorithm seems to result in the vast majority of nodes joining a channel and reaching their capture ratio, with little time wasted on other processes.

Convergence analysis of the proposed algorithm is part of ongoing research. Future plans include the use of relay nodes in the network that will allow for a more balanced sharing of the channels between the nodes.

\section{REFERENCES}

[1] E.-S. Jung and N. H. Vaidya. A power control MAC protocol for ad hoc networks. In MobiCom '02: Proceedings of the 8th annual international conference on Mobile computing and networking, pages 36-47, New York, NY, USA, 2002. ACM.

[2] T. ElBatt and A. Ephremides. Joint Scheduling and Power Control for Wireless Ad-hoc Networks. In Proceedings of IEEE INFOCOM, 2002.

[3] J. Tang, G. Xue, C. Chandler, and W. Zhang. Link scheduling with power control for throughput enhancement in multihop wireless networks. In Proceedings of the Second International Conference on Quality of Service in Heterogeneous Wired/Wireless Networks, page 1, Washington, DC, USA, 2005. IEEE Computer Society.

[4] Sastry Kompella, Jeffrey E. Wieselthier, and Anthony Ephremides. A Cross-layer Approach to Optimal Wireless Link Scheduling with SINR Constraints. In MILCOM, 2007.

[5] Y. Li and A. Ephremides. A joint scheduling, power control, and routing algorithm for ad hoc wireless networks. Ad Hoc Netw., 5(7):959-973, 2007.

[6] Bastian Katz, Markus Volker, and Dorothea Wagner. Energy efficient scheduling with power control for wireless networks. In Modeling and Optimization in Mobile, Ad Hoc and Wireless Networks (WiOpt), 2010 Proceedings of the 8th International Symposium on, pages 160 -169, June 2010.

[7] Nicholas Bambos, Shou C. Chen, and Gregory J. Pottie. Channel Access Algorithms with Active Link Protection for Wireless Communication Networks with Power Control. IEEE/ACM Transactions on Networking, 8(5):583-597, 2000.

[8] Q. Wu. Optimum transmitter power control in cellular systems with heterogeneous sir thresholds. IEEE Transactions on Vehicular Technology, 49(4):1424-1429, July 2000.

[9] S. Dontula and S. Jagannathan. Active link protection for wireless peerto-peer and cellular networks with power control. In Proceedings of the World Wireless Congress, pages 612-617, 2004.

[10] Roger A. Horn and Charles R. Johnson. Matrix Analysis. Cambridge University Press, 1985.

[11] Roger A. Horn and Charles R. Johnson. Topics in Matrix Analysis. Cambridge University Press, 1994.

[12] G. Foschini and Z. Miljanic. A Simple Distributed Autonomous Power Control Algorithm and its Convergence. IEEE Transactions on Vehicular Technology, 42(4):641-646, November 1993.

[13] Muhammad Hamad Alizai, Olaf Landsiedel, Jó Ágila Bitsch Link, Stefan Götz, and Klaus Wehrle. Bursty traffic over bursty links. In Proceedings of the 7th ACM Conference on Embedded Networked Sensor Systems, SenSys '09, pages 71-84, New York, NY, USA, 2009. ACM. 